\title{
XerR, a negative regulator of XccR in Xanthomonas campestris pv. campestris, relieves its repressor function in planta
}

\author{
Li Wang ${ }^{1,2,3}$, Lili Zhang ${ }^{4}$, Yunfeng Geng ${ }^{5}$, Wei Xi ${ }^{1}$, Rongxiang Fang ${ }^{1,2}$, Yantao Jia ${ }^{1,2}$ \\ ${ }^{I}$ State Key Laboratory of Plant Genomics, Institute of Microbiology, Chinese Academy of Sciences, No. 3 Yard 1 West Beichen \\ Road, Chaoyang District, Beijing 100101, China; ${ }^{2}$ National Plant Gene Research Center, Beijing 100101, China; ${ }^{3}$ Graduate \\ School of the Chinese Academy of Sciences, Beijing 100049, China
}

We previously reported that XccR, a LuxR-type regulator of Xanthomonas campestris pv. campestris (Xcc), activates the downstream proline iminopeptidase virulence gene (pip) in response to certain host plant factor(s). In this report, we further show that the expression of the $x c c R$ gene was repressed in the culture medium by an NtrCtype response regulator, which we named XerR ( relieved when the bacteria were grown in planta. Such a regulatory mechanism is reinforced by the observations that XerR directly bound to the $x c c R$ promoter in vitro, and that mutations at the phosphorylation-related residues of XerR resulted in the loss of its repressor function. Furthermore, the expression level of $x c c R$ increased even in XerRoverexpressing $X c c$ cells when they were vacuum infiltrated into cabbage plants. We also preliminarily characterized the host factor(s) involved in the above mentioned interactions between $X c c$ and the host plant, showing that a plant material(s) with molecular weight(s) less than $1 \mathrm{kDa}$ abolished the binding of XerR to the $x c c R$ promoter, while the same material enhanced the binding of XccR to the luxXc box in the pip promoter. Taken together, our results implicate XerR in a new layer of the regulatory mechanism controlling the expression of the virulence-related $x c c R / p i p$ locus and provide clues to the identification of plant signal molecules that interact with XerR and XccR to enhance the virulence of $X c c$.

Keywords: Xcc; NtrC-like regulator; LuxR-like regulator; proline iminopeptidase; pathogen-host interaction; plant signal(s) Cell Research (2011) 21:1131-1142. doi:10.1038/cr.2011.64; published online 12 April 2011

\section{Introduction}

In the past few decades it has become obvious that bacteria can display sophisticated group behaviors and form communities in their natural niches in response to constant changes in physical, chemical and biological environments [1-3]. The regulation of gene expression mediated by signaling molecules and regulatory proteins

Correspondence: Yantao Jia ${ }^{\mathrm{a}}$, Rongxiang Fang ${ }^{\mathrm{b}}$

${ }^{\mathrm{a}}$ Tel: +86-10-64861838, Fax: +86-10-64858245

E-mail: jiayt@im.ac.cn

${ }^{\mathrm{b}} \mathrm{Tel} / \mathrm{Fax}$ : +86-10-64858245

E-mail: fangrx@im.ac.cn

${ }^{4}$ Current address: Department of Internal Medicine, Yale School of Medicine, New Haven, CT 06510, USA

${ }^{5}$ Current address: Temasek Life Sciences Laboratory, 1 Research Link, The National University of Singapore, Singapore 117604, Singapore

Received 31 May 2010; revised 12 August 2010; accepted 18 November 2010; published online 12 April 2011 in a bacterial population density-dependent manner is referred to as quorum sensing (QS). The first QS system in Gram-negative bacteria was observed in Vibrio fischeri, which contains a LuxR regulator and a cognate LuxI synthase responsible for producing autoinducer signal molecules $N$-acylhomoserine lactones (AHLs) [4-6]. To date, QS-dependent functions have been studied in a wide variety of bacteria that control diverse bacterial processes, including virulence, sporulation, plasmid transfer, biosynthesis of antibiotics, as well as plant nodulation [7-9]. It is now increasingly evident that QS is a complicated group behavior of bacteria for producing, sensing and responding to multifarious chemical signals to increase their chances of survival and propagation [7, 8]. In other cases, QS-mediated communications are also involved in interactions between bacterial species and between bacteria and their hosts. For example, $\gamma$-amino butyric acid (GABA) produced by plant induces the expression of the attKLM operon in Agrobacterium tumefaciens, 
which causes the bacterium to destroy its own QS signal [10], while L-proline interferes with the import of GABA and antagonizes the degradation of bacterial QS signal, 3-oxo-octanoylhomoserine lactone [11].

A genomic survey of Proteobacteria showed that there are numerous bacteria that do not encode a cognate LuxI synthase for AHLs [12]. As a result, the unpaired LuxR-like proteins designated as LuxR-family orphans or 'solos' have been studied [13, 14]. LuxR solos such as ExpR of Sinorhizobium meliloti, BisR of Rhizobium leguminosarum pv. viciae and QscR of Pseuodomonas aeruginosa, respond to AHL signals produced by the bacteria themselves [15-17]. In addition, SdiA in Salmonella, Escherichia and Klebsiella are able to bind and detect AHLs produced by other bacterial species [18]. Interestingly, accumulating evidence from recent studies supports the idea that, apart from playing important roles in sensing AHL-like autoinducers, LuxR-like solos could potentially sense non-AHL signaling molecules as well $[13,14,19]$.

As a special LuxR-like solo, XccR of the plant pathogen Xanthomonas campestris pv. campestris (Xcc) is required for activating the expression of the downstream proline iminopeptidase gene (pip) through binding to the luxXc box in the pip promoter, and this activation is enhanced by plant host factors [20]. The $x c c R / p i p$ locus is different from the classical luxR/luxI system in that pip is a virulence-related gene, rather than a gene for producing AHL signals. The $x c c R / p i p$-like locus has been found in several other bacteria, such as $S$. meliloti, Rhodospirillum rubrum, $R$. leguminosarum and P. syringae [20]. More particularly, the oryR/pip locus of Xanthomonas oryzae pv. oryzae (Xoo) behaves very much like the $x c c R / p i p$ locus. In addition, the solubility of OryR is enhanced by a rice extract with molecular weights less than $1 \mathrm{kDa}$ [21]. OryR also positively regulates the expression of a cell wall-degrading cellobiosidase gene for optimal pathogenicity [22].

In this study, we explored the bacterial upstream factor(s) and the host plant signals regulating the expression of the $x c c R / p i p$ locus. By screening a genomescale Tn5-insertion library of an Xcc strain harboring an $x c c R$ promoter-gusA fusion, we identified an NtrC-type transcriptional regulator XC 3760 (named XerR, XccR expression-related, repressor) as a repressor of the $x c c R /$ pip locus. NtrC-type proteins have been recognized as enhancer-binding proteins in phosphorylated forms; they are involved in nitrogen assimilation, biofilm formation, bioluminescence and QS regulatory system, and thus their functions are expected to be pleiotropic [23-26]. Furthermore, we showed that the repressor function of XerR was relieved in the presence of the host plant ex- tract with molecular weights less than $1 \mathrm{kDa}$, and that the same plant extract enhanced the binding of XccR to the pip promoter sequence. Our results expand the regulatory machinery controlling the expression of the pathogenicity-related $x c c R /$ pip locus and provide new insights into how $X c c$ senses host signals to regulate its infectivity.

\section{Results}

Genetic screening of xccR expression reveals a repressor, XerR

To identify factors that regulate the expression of $x c c R$, which directs the expression of the virulence gene pip in $X c c$, we designed an antibiotic-coupled transposon screen. The chromosomal $x c c R$ promoter $(x c c R-\mathrm{P}) /$ gusA fusion strain (Xcc 8177) was mutated with the EZTn5 transposon that contains the dihydrofolate reductase (DHFR) gene for conferring trimethoprim resistance. From 20000 transposon-insertion mutants, we selected one that pointed to a possible repressor of $x c c R$ expression. Analysis of the flanking sequences of the mutated gene indicated the gene was $X C \_3760$ encoding a transcriptional regulator of the NtrC family, which we designated as $x e r R$ in this paper.

XerR is a putative 433 amino acid protein with a predicted molecular weight of $48.2 \mathrm{kDa}$ and belongs to the two-component signal transduction system (TCSTS) response regulator (RR) NtrC family [27]. BLAST search against the databases revealed that XerR is highly conserved in all Xanthomonas species and shares significant sequence similarities with $\mathrm{NtrC}$ family proteins from Bordetella bronchiseptica (61\%), Ralstonia solanacearum $(60 \%)$ and Burkholderia xenovorans $(58 \%$; NCBI Blast: http://www.ncbi.nlm.nih.gov/BLAST/). Analyses with Pfam indicated that XerR contains an N-terminal receiver domain that has the conserved Asp site for phosphorylation, a central ATP-binding AAA + domain that hydrolyzes ATP to generate energy, and a C-terminal domain containing a helix-turn-helix motif for DNA binding [23]. Multiple sequence alignments between XerR and CheY in Escherichia coli [28], NtrC in Salmonella typhimurium [29] and LuxO in Vibrio harveyi [25], revealed the highly conserved residues Asp-17, Asp-60 and Phe-106 in the receiver motif (Figure 1A). These residues were shown to be critical for the functioning of the phosphorylated protein, and Asp-60 was proposed to be the phosphorylated site. Although the histidine kinase and its cognate RR are usually linked in one operon [30], a search of the genome did not suggest that the Xcc chromosome encodes a cognate TCSTS sensor protein in close vicinity of the $x e r R$ sequence. It was the overall structure and location of $x e r R$ that prompted us to study 
A

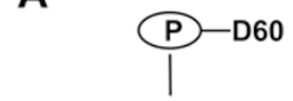

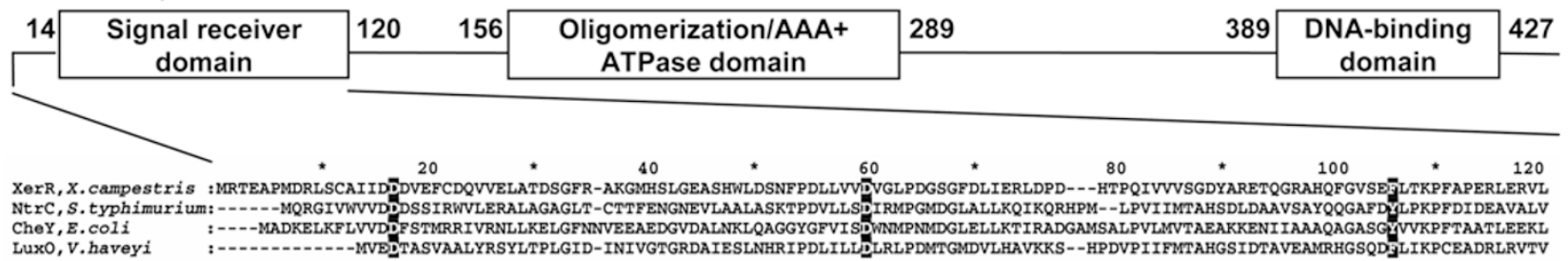

B

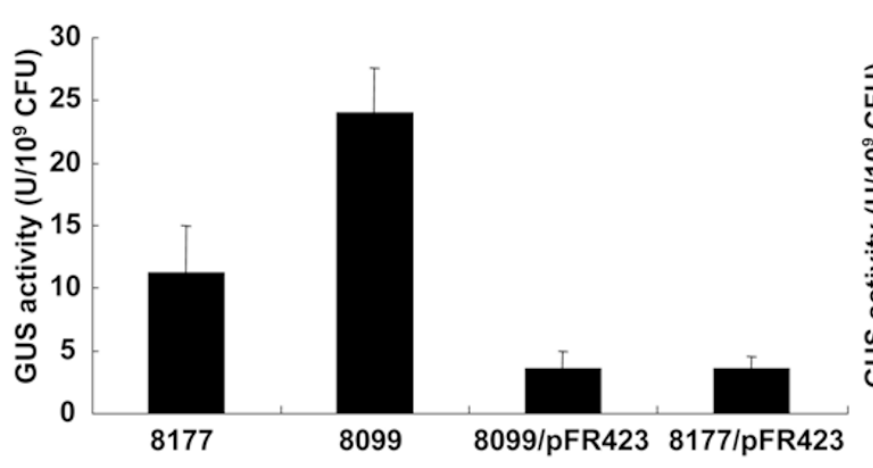

D
C

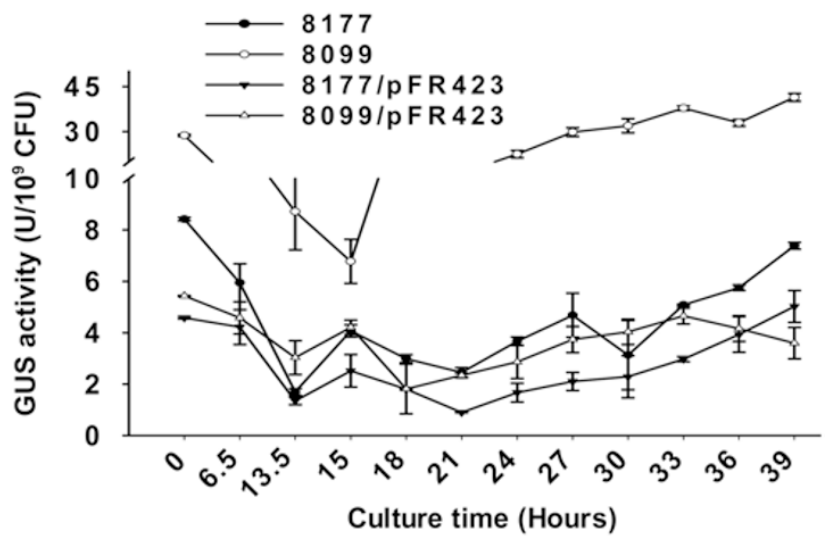

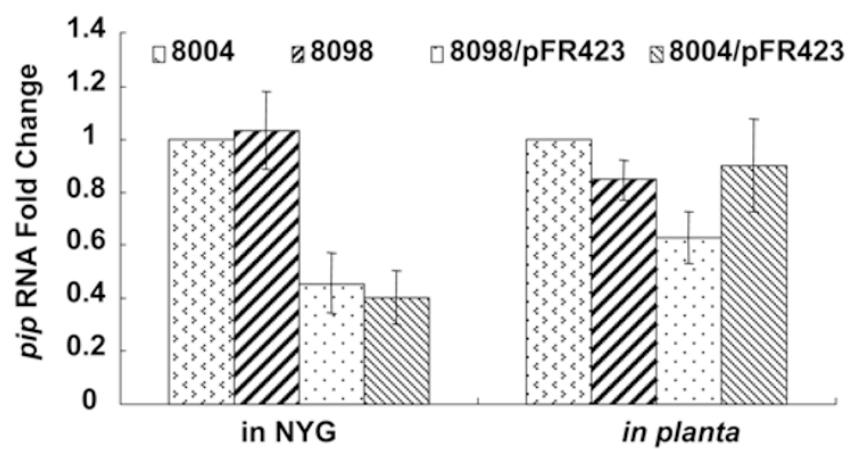

Figure 1 XerR is required for repression of $x c c R$ and pip transcription in medium. (A) The domain organization of $\mathrm{XerR}$ and the sequence of receiver domain. Three putative modular components of XerR are shown in the diagram. Multiple amino acid sequence alignments between XerR and NtrC in S. typhimurium, CheY in E. coli and LuxO in V. harveyi are shown at the bottom of the diagram. The residues altered by site-directed mutagenesis are shaded in black, and the putative phosphorylation site (Asp-60) is marked. (B) GUS expression levels in different Xcc strains were assayed by enzymatic activities. xerR in-frame deletion mutant Xcc 8099 increased the GUS activity compared to that of Xcc 8177 . Xcc 8099 and Xcc 8177 carrying the xerR gene in pHM1 plasmid (Xcc 8099/pFR423 and Xcc 8177/pFR423) exhibited reduced GUS activities. All the strains were harvested at $\mathrm{OD}_{600}$ of 2.0 in NYG medium. Relative GUS activity units were defined as nM 4-methylumbelliferyl $/ \mathrm{min} / 10^{9}$ cells. The means and standard deviations were calculated from the data derived from at least nine independent experiments. (C) Expression of $x c c R-P / g u s A$ in Xcc 8099 was density dependent when grown in NYG medium. GUS activities of different strains were assayed at different time points. The mean and standard deviation were calculated from the data derived from three independent experiments. (D) Expression levels of pip in different Xcc strains in medium and in planta. Relative transcriptional levels of pip were quantified by real-time RT-PCR. In NYG medium, RNA were extracted from the cultured strains at a cell density of $\mathrm{OD}_{600}=1.5-2.0$. In planta, RNA was isolated from vacuum-infiltrated cabbage leaves $30 \mathrm{~h}$ post infiltration. Measurements were normalized by the wild-type values and fold differences were plotted. Each sample was assayed in triplicate. 
its biological functions.

To verify the role of XerR in $x c c R$ repression, an $x e r R$ non-polar markerless deletion was introduced into the chromosome of Xcc 8004 to generate the xerR mutant $X c c$ 8098, and then an $x c c R-\mathrm{P} / g u s A$ fusion was inserted into Xcc 8098 to create $X c c$ 8099. $\beta$-Glucuronidase (GUS) activities in $X c c$ 8099, its complementation strain $X c c$ 8099/pFR423, in which pFR423 carries xerR driven by the $l a c Z$ promoter, and the XerR-overexpression strain Xcc $8177 /$ pFR423 were examined and compared with that in Xcc 8177 at mid-exponential phases of bacterial growth (Figure 1B). The results showed that GUS activity in Xcc 8099 was increased 2.14-fold relative to Xcc 8177, whereas the GUS activities in both complementation and overexpression strains were reduced to $30 \%$ of that of Xcc 8177. Although GUS activities in Xcc 8177 did not display a typical QS behavior and stayed at low levels as those in Xcc 8099/pFR423 and Xcc 8177/ pFR423, GUS levels in Xcc 8099 increased along with the cell growth (Figure 1C). These data suggest that derepressed expression of $x c c R$ occurs in a density-dependent manner.

We previously showed that under medium culture conditions, overexpression of XccR significantly enhanced the expression of the downstream pip gene, while in wild-type Xcc 8004 pip expression remained very low throughout the bacterial growth phases. Here we tested whether the XerR protein has an indirect effect on pip transcription. By using real-time reverse transcription (RT)-PCR, we found that in XerR-overexpression strains Xcc 8098/pFR423 and Xcc 8004/pFR423, the level of the pip transcript decreased significantly by $55 \%$ and $60 \%$, respectively (Figure 1D). One possibility is that XerR also potentially repressed the expression of pip. However, the pip RNA levels in xerR-deleted Xcc 8098 showed little increase compared with that in Xcc 8004. Furthermore, when Xcc 8004 and Xcc 8098 were grown in NYG medium, no XccR protein was detected with anti-XccR antibodies (data not shown). The result is similar to OryR protein, a homolog of XccR in Xoo, which was also not detectable by western blot analysis when bacteria were grown in minimal M9 medium [21]. These results indicate that the increased expression of $x c c R$ by $x e r R$ mutation was not sufficient to provide enough stable XccR protein to alter the pip RNA level.

$X e r R$ acts as a repressor by binding to the $x c c R$ promoter

Usually two-component RR NtrC family proteins act on phosphorylation as enhancer-binding proteins via interaction with $\sigma^{54}$. However, analysis of the $x c c R$ promoter sequence did not reveal a highly conserved $\sigma^{54}$ recognition sequence GG-N $\mathrm{N}_{10}-\mathrm{GC}$ [31]. We thus explored the possibility that XerR directly interacts with the $x c c R$ promoter. Electrophoretic mobility shift assays (EMSAs) were performed using purified XerR protein tagged with an N-terminal MBP and DNA sequences upstream of the $x c c R$ coding region as probes, which spanned -50 to +9 (R1) and -99 to -40 (R2), respectively, relative to the translational start site (Figure 2A). Addition of MBPXerR to the reaction mixtures caused a shift in the mobility of R1 fragment (Figure 2B) and R2 fragment (Figure $4 \mathrm{C})$, but addition of pure MBP did not. The shifted bands could be competed by 50 -fold excess of the unlabeled probes, indicating a specific binding of XerR to the $x c c R$ promoter. The binding affinity of XerR affirms its ability to repress the $x c c R$ transcription, and as a repressor it likely prevents RNA polymerase from binding to tran-

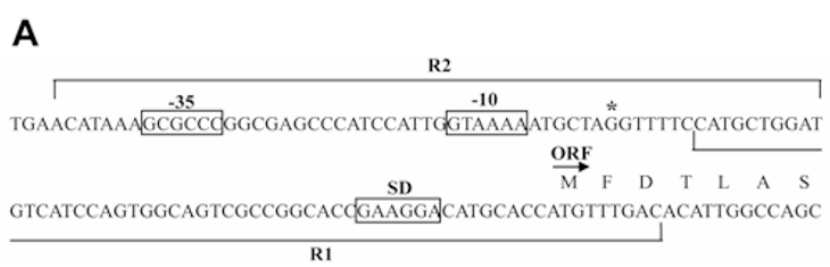

B

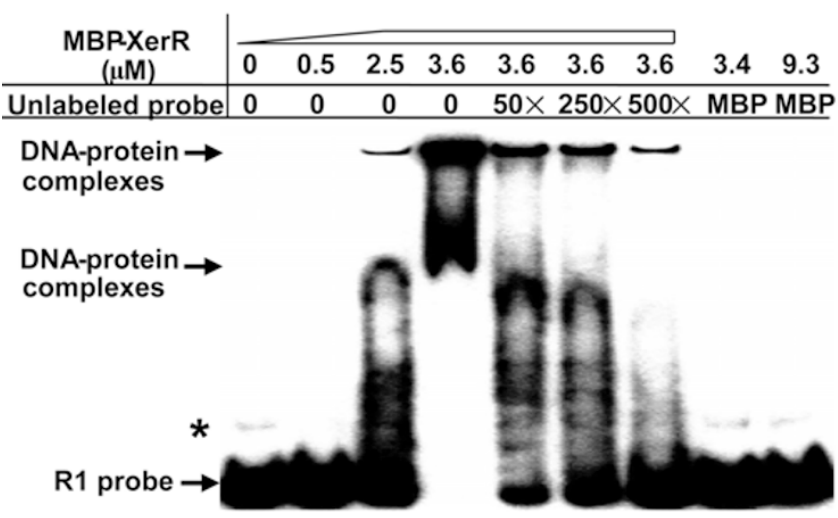

Figure 2 EMSA shows that XerR binds to the upstream region of the $x c c R$ gene directly. (A) Schematic of the upstream region of $x C c R$ gene according to a promoter prediction program NNPP version 2.2 (1999). The putative $-35 /-10$ and SD (ShineDalgarno sequence) elements are boxed, and an asterisk denotes the $x c c R$ transcriptional start site. The locations of two different probes that have a 10-bp overlap are denoted by lines. (B) EMSA assay of R1 probe with purified MBP-XerR. Isotopelabeled probe $(8 \mathrm{fmol})$ was incubated for $30 \mathrm{~min}$ with indicated concentrations of protein (in $\mu \mathrm{M}$ ) at room temperature. The shifted bands could be competed by excess of the unlabeled probe. The folds of unlabeled probe were indicated above. The migrated DNA-protein complexes and free probe R1 are indicated by arrows, and the bands marked with an asterisk indicate a possible higher structure of R1 probe formed during annealing step. 
scriptional sites and ensures that the gene is turned off in an efficient and specific manner [32, 33].

Phosphorylation-related residues of XerR are essential for its repressor function

In vivo and in vitro experiments indicated that XerR can efficiently repress the expression of $x c c R$. To characterize the repressor function-related motifs, we first tested whether the $\mathrm{N}$-terminal part or the $\mathrm{C}$-terminal part of XerR is critical in regulating transcription of $x c c R$. We constructed an xerR RR domain (N-terminal amino acids 14-120) deletion mutant (xerR $\Delta \mathrm{RR}$ ) and an HTH domain (C-terminal amino acids 385-433) deletion mutant (xerR $\Delta \mathrm{HTH}$ ), and assayed GUS activities under the control of the $x c c R$ promoter in these two $x e r R$ deletion strains. The results showed that the GUS activities produced by xer $R$ $\Delta \mathrm{RR}$ and $x e r R \Delta \mathrm{HTH}$ were 3.06 times and 2.97 times, respectively, of that of Xcc 8177 in NYG medium (Figure $3 \mathrm{~A}$ ), indicating that removal of the receiver or DNAbinding domain results in an inactive XerR protein and
A

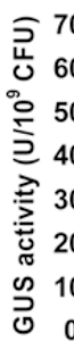

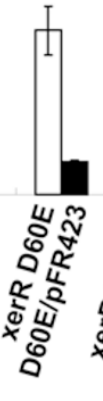

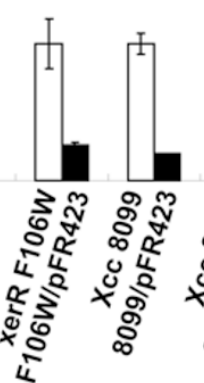

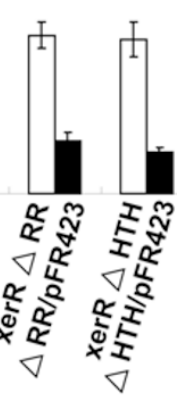

B

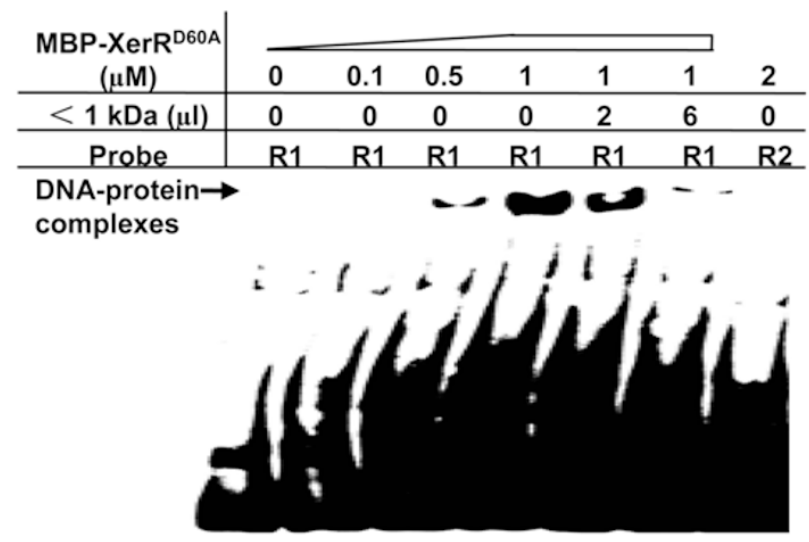

D
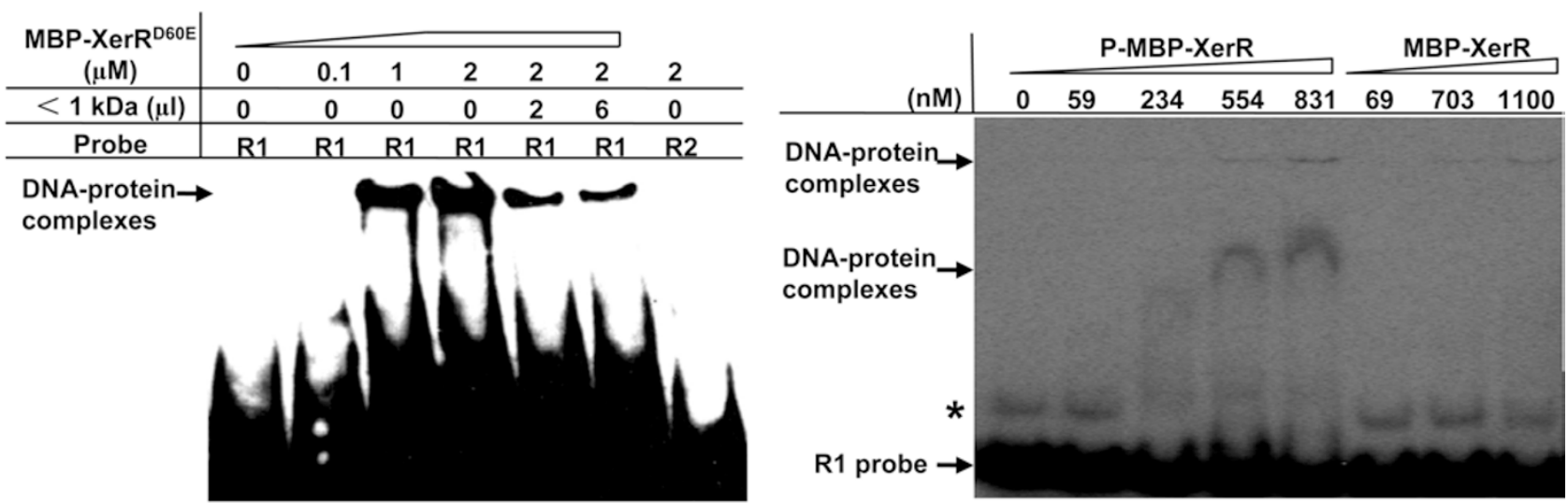

Figure 3 Phosphorylation-related residues of XerR are essential for its repressor function. (A) The conserved phosphorylation-related residues and the regulatory domains of XerR were indispensable for regulation of $x c c R$ expression in vivo. xerR $\triangle \mathrm{RR}$, xerR $\triangle \mathrm{HTH}$ and different site-directed mutants exhibited increased GUS activities when grown in NYG medium. Plasmid-containing (pFR423) strains of different mutants reduced the GUS activities compared with that of Xcc 8177 . Bacteria cultured in NYG medium were assayed at an $\mathrm{OD}_{600}$ of 2.0. The experiments were repeated eight times with similar results. (B and C) EMSA assays of MBP-XerR ${ }^{\mathrm{D} 60 \mathrm{~A}}$ and MBP-XerR ${ }^{\mathrm{D} 60 \mathrm{E}}$ with biotin-labeled $\mathrm{R} 1$ probe and plant extract. The two proteins presented the same binding characteristics to $\mathrm{R} 1$ probe, in which the plant extract of molecular weights $<1 \mathrm{kDa}$ released the protein and DNA interactions. In the diagram, the concentration of purified protein and the volumes of plant signal(s) are indicated. (D) EMSA binding of phosphorylated and unphosphorylated MBP-XerR to the R1 probe. MBP-XerR was phosphorylated in vitro with acetyl phosphate and the R1 probe was end-labeled with ${ }^{32} \mathrm{P}$ at its $5^{\prime}$ termini. The bands marked with an asterisk indicate a possible higher structure of R1 probe formed during annealing step. 
thus de-represses the $x c c R$ transcription.

Next, we investigated the roles of the phosphorylation-related residues Asp-17, Asp-60 and Phe-106 in the repressor function of XerR. We constructed four xerR site-directed mutants on the Xcc 8177 background (xerR D17K, xerR D60A, xerR D60E and xerR F106W) and assayed their GUS activities (Figure 3A). Similar to the xerR null mutant Xcc 8099, each site-directed mutant showed a considerably higher GUS level than Xcc 8177, suggesting that the canonical phosphorylation-related residues are required for XerR function in vivo. Furthermore, the low-copy plasmid carrying wild-type xerR (pFR423) was able to restore the XerR repressor activity in trans in all of the xerR deletion and site-directed mutants (Figure 3A).

In addition, we analyzed whether the Asp-60-mutated proteins MBP-XerR ${ }^{\mathrm{D} 60 \mathrm{~A}}$ and MBP-XerR ${ }^{\mathrm{D} 60 \mathrm{E}}$ can still bind the $x c c R$ promoter sequences, since Asp-60 was proposed to be the phosphorylation site by Pfam alignment. We found that although both mutated proteins could bind to $\mathrm{R} 1$, they lost the ability to bind $\mathrm{R} 2$ even at higher protein concentrations (Figure 3B and 3C), suggesting that Xe$\mathrm{rR}^{\mathrm{D} 60 \mathrm{~A}}$ and $\mathrm{XerR}^{\mathrm{D} 60 \mathrm{E}}$ have altered DNA binding properties and thus cannot repress the $x c c R$ promoter.

On the other hand, we found that phosphorylation of XerR enhanced the binding to R1. As shown in Figure $3 \mathrm{D}$, in vitro phosphorylated XerR (P-MBP-XerR) exhibited an affinity to bind R1 in EMSA at a concentration of $554 \mathrm{nM}$, which is lower than that needed for unphosphorylated MBP-XerR protein. Under an equivalent condition, we did not observe the band-shift at $1.1 \mu \mathrm{M}$ for unphosphorylated MBP-XerR protein.

Taken together, the above results indicate that phosphorylation of XerR is essential for its repressor function, reminiscent of the intrinsic property of an $\mathrm{NtrC}$ family protein.

\section{Inhibition of xccR expression by XerR is relieved in planta}

We previously reported that the expression of $x c c R$ and pip was induced when the $X c c$ cells grew in the host cabbage [20]. In this report, we showed that XerR inhibited the expression of $x c c R$ and pip in culture medium. To see if the XerR-mediated inhibition is affected in planta, we quantified and compared the $x c c R$ expression levels in planta (Figure 4A) and in NYG medium (Figure 1B) in different $X c c$ strains. Overexpression of XerR in Xcc $8099 / \mathrm{pFR} 423$ and $X c c 8177 / \mathrm{pFR} 423$ greatly reduced the $x c c R$ promoter-directed GUS activities in NYG medium compared with that of $X c c$ 8177, as shown in Figure 1B. However, the GUS activities in the XerR-overexpressing strains were not reduced or even increased relative to that of $X c c 8177$ when the bacteria grew in planta (Figure

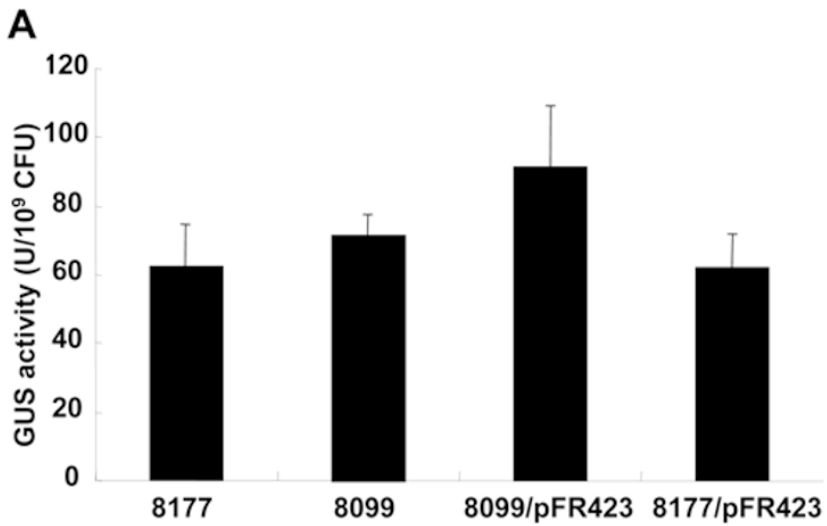

B

\begin{tabular}{|c|c|c|c|c|c|}
\hline \multirow{2}{*}{$\begin{array}{c}\text { MBP-XerR } \\
(\mu \mathrm{M})\end{array}$} & \multicolumn{5}{|c|}{ 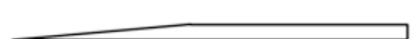 } \\
\hline & $\overline{0}$ & 1 & 2 & 2 & 2 \\
\hline$<1 \mathrm{kDa}(\mu \mathrm{l})$ & 0 & 0 & 0 & 5 & 13 \\
\hline
\end{tabular}

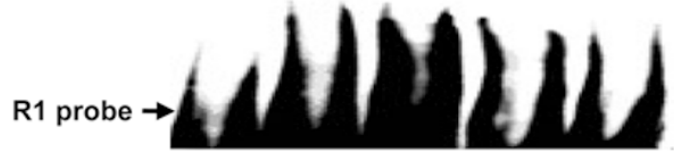

C
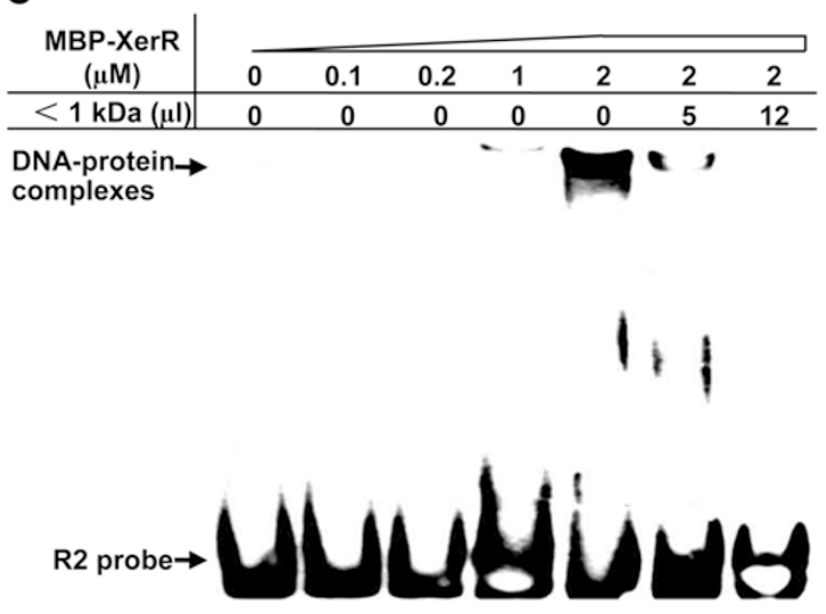

Figure 4 XerR relieves its inhibition on $x c c R$ expression in planta. (A) In planta cultivation did not significantly increase the GUS activity from Xcc 8099, while it had the opposite influence on that of Xcc 8099/pFR423 and Xcc 8177/pFR423. The bacteria were recovered from vacuum-infiltrated cabbage leaves 30 h post infiltration, and GUS activities were assayed. Data and standard deviation represented the mean of three independent measurements. (B and C) Plant signal(s) alleviated the binding activity of XerR protein to the $x c c R$ upstream regulatory sequence. EMSA assays with biotin-labeled probe were performed by MBP-XerR with plant extracts $(<1 \mathrm{kDa})$ at two dilutions. 
4A). Furthermore, the Xcc 8099 strain had almost equivalent GUS activity as Xcc 8177 (Figure 4A), suggesting that the repression action of XerR on $x c c R$ expression might be relieved in planta.

As pip expression is controlled by $\mathrm{XccR}$, we expected the inhibition of expression of pip by XerR in culture medium would also be relieved in planta. This was actually the case. As seen in Figure 1D, the significantly reduced pip transcript levels in XerR-overexpressing strains Xcc 8098/pFR423 and Xcc 8004/pFR423 in culture medium were restored to $63 \%$ and $90 \%$ of that of wild-type Xcc 8004, respectively, when the bacteria grew in planta. We reasoned that the increased expression of $x c c R$ and pip was not a result of reduced transcription of $x e r R$ gene in host plant, because the GUS activity of $x e r R-\mathrm{P} / g$ us $A$ in planta was 2.56 -fold higher than that in medium alone (data not shown). In addition, the expression of $X C_{-} 3756$, another gene that is directly regulated by XerR via binding to the $\sigma^{54}$ cis-element in its promoter, was enhanced threefold in planta compared with that in medium (data not shown).

To examine whether the observed de-repression of $x c c R$ expression was caused by plant factors that affected the binding of XerR to the $x c c R$ regulatory sequences, we performed EMSA in the presence of a low-molecularweight $(<1 \mathrm{kDa})$ cabbage extract. As shown in Figure $4 \mathrm{~B}$ and $4 \mathrm{C}$, the presence of the plant extract disrupted the binding of XerR to the $x c c R$ promoter probes $\mathrm{R} 1$ and R2 in a dose-dependent manner. We infer from this result that some small molecules present in this cabbage extract are responsible for limiting the XerR binding to DNA. This interfering effect was DNA sequence specific, as the same amount of the cabbage extract did not cause dissociation of XerR from the promoter of another downstream gene $X C \_3756$ (data not shown). We further showed that the same extract also abrogated the ability of the mutant proteins MBP-XerR ${ }^{\mathrm{D} 60 \mathrm{~A}}$ and MBP-XerR ${ }^{\mathrm{D} 60 \mathrm{E}}$ to retard the migration of the DNA probe R1 (Figure 3B and $3 \mathrm{C}$ ), indicating that the two amino acid substitutions for Asp-60 of XerR do not alter the interaction between XerR and the plant signal(s).

\section{Cabbage extract enhances the binding of XccR to the pip promoter}

We previously reported that in a super-shift assay, a cabbage ethanol extract enhanced the binding of XccR to the pip promoter [20]. In addition, it was reported that an unknown rice signal molecule present in the $<1 \mathrm{kDa}$ fraction of an Xoo-infected rice extract increased the solubility of OryR [21]. We thus wanted to see if the < $1 \mathrm{kDa}$ cabbage extract, which abolished the binding of XerR to the $x c c R$ promoter, could affect the binding of

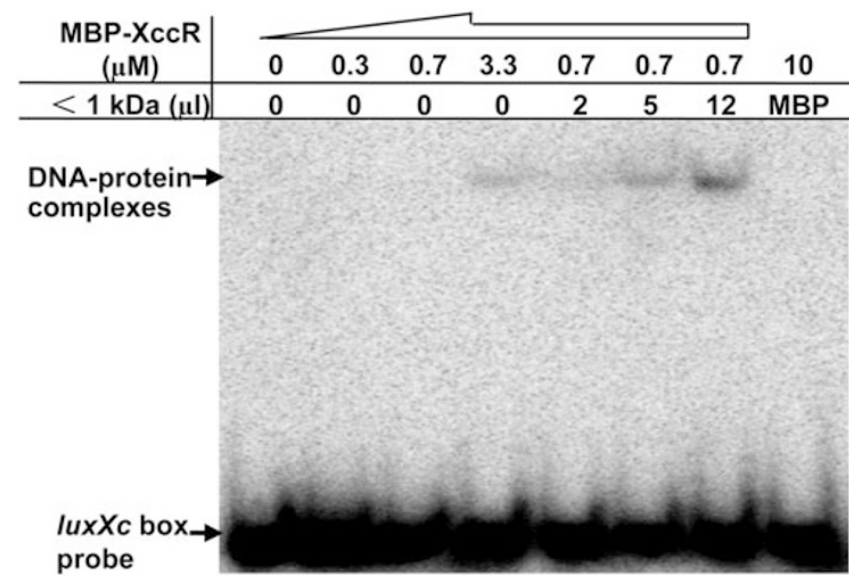

Figure 5 EMSA binding of MBP-XccR protein to the luxXc box of the pip promoter. The band of XccR and DNA complex was intensified by adding different volumes of plant extracts in the EMSA assay. The migrated DNA-protein complexes and isotope-labeled luxXc box probe are indicated by arrows.

XccR to the luxXc box of the pip promoter. We found that the $<1 \mathrm{kDa}$ extract indeed stimulated the binding of MBP-XccR to the luxXc box sequence at an MBP-XccR concentration of $0.7 \mu \mathrm{M}$ at which no protein-DNA binding occurred without the plant extract (Figure 5). Moreover, the formed protein-DNA complex was significantly intensified with an increase in the concentration of the plant extract (Figure 5). The above results indicate that the same cabbage extract shows different effects on the formation of the XerR/xccR promoter complex and the $\mathrm{XccR} /$ pip promoter complex. Although we cannot conclude that XerR and XccR interact with the same compound in the cabbage extract, our results portray a subtle regulatory pattern in which $X c c$ recruits plant signal(s) to sequester XerR from its binding sequence, yet on the other hand, to stimulate the XccR binding to the pip promoter for infectivity

\section{Discussion}

In Gram-negative bacteria, LuxR/LuxI is the most well-defined regulatory system that modulates gene expression related to QS. This system can monitor the concentration of AHL-like small molecules in the environment and control downstream gene expression or cell behavior [34, 35]. In Xanthomonas, the solo (orphan) LuxR homologs, including XccR from Xcc and OryR from $X o o$, can sense chemical signals derived from host plants and take part in bacterial pathogenesis by regulating the expression of virulence factors [20-22]. These studies strongly suggested an interesting phenomenon 
that an inter-kingdom communication exists between phytopathogens and their host plants. However, how bacteria sense signals from plant and the nature of the plant signal(s) remain unclear.

In this study, an NtrC family RR was identified by genome-scale screening with a transposon insertional mutant library. By measurement of GUS activity, XerR was confirmed to be a negative regulator of $x c c R$ expression (Figure 1B). XerR can bind R1 and R2 regions of $x c c R$ upstream sequence (Figures $2 \mathrm{~B}$ and $4 \mathrm{C}$ ). It was shown that mutations of the conserved phosphorylationrelated sites on XerR resulted in upregulation of $x c c R$ expression (Figure 3A) and that in vitro phosphorylated XerR showed enhanced affinity to the R1 probe (Figure 3D), both results suggesting that protein phosphorylation is required for the repression function of XerR. In addition, GUS assays in planta (Figure 4A) as well as EMSA experiments (Figure 4B and 4C) showed that the binding of XerR to the $x c c R$ upstream DNA sequence was substantially inhibited in the presence of the plant extract, suggesting that a plant signal(s) modulates the xerR/ $x c c R /$ pip regulatory cascade.

By secondary protein structure prediction (Figure 1A), XerR was found to be a typical NtrC-family RR of the bacterial TCSTS. It contains an N-terminal CheYlike receiver domain, a $\mathrm{C}$-terminal $\mathrm{HTH}$ domain, and a central $\sigma^{54}$ interaction domain responsible for the initiation of an open transcriptional complex [36]. In prototypical TCSTS, a histidine kinase (HK) sensor can monitor specific environmental stimuli. After autophosphorylation on a conserved histidine residue, the dimeric HK transfers the phosphoryl group onto the conserved asparagic acid residue of the cognate $\mathrm{RR}$, and the latter will regulate downstream gene expression, usually acting as a transcription factor [37, 38]. As mentioned above, site-directed mutations, which changed the three critical sites (Asp-17, Asp-60 and Phe-106) related to protein phosphorylation, nearly abolished the repressor activity of XerR (Figure 3A). In addition, XerR can receive the phosphorylation signal in vitro (Figure 3D). These results suggest that under the culture conditions, an unidentified HK can phosphorylate XerR specifically and cause it to bind to the $x c c R$ promoter with high affinity. Transcriptional repression of $x c c R$ may result from the direct competition of phosphorylated-XerR and RNA polymerase on its promoter or the blocking of mRNA elongation, as the probes that we used spanned from -99 to $+9 \mathrm{bp}$ relative to the $x c c R$ translational start site. The repression mechanism of XerR remains to be fully elucidated. Intriguingly, $\mathrm{XerR}^{\mathrm{D} 60 \mathrm{~A}}$ and $\mathrm{XerR}^{\mathrm{D} 60 \mathrm{E}}$ abolished the binding ability to R2 (Figure 3B and 3C), whereas the $E$. coli $\mathrm{NtrC}^{\mathrm{D} 60 \mathrm{~A}}$ and $\mathrm{NtrC}^{\mathrm{D} 60 \mathrm{E}}$ proteins bound to the cognate enhancer sequence normally $[39,40]$.

In addition, our results also showed that the repressor function of XerR was relieved when $X c c$ was grown within the host plant (Figure 4A). In TCSTS, the central mechanism is the balance of phosphorylated and dephosphorylated RR [41, 42]. Since the majority of HKs also have phosphatase activity to dephosphorylate RR [43-45], we speculated that the cognate HK of XerR in planta may exhibit phosphatase activity, rather than acting as a kinase, which would result in the dephosphorylation of XerR and thus decrease its affinity in binding the $x c c R$ promoter. Because there is no HK gene located in the vicinity of $x e r R$, it is extremely difficult to identify its cognate HK only by bioinformatic analysis. Currently we have identified a candidate HK gene that also exhibits repressor function in regulating $x c c R$ transcription (unpublished data). Further investigation will clarify whether it is the cognate HK of XerR and how it modulates the phosphorylation state of downstream RR when Xcc survives in different ecological niches.

Besides phosphorylation-regulated $x c c R$ expression via classical TCSTS, our experiments also suggest that the activity of XerR is affected by chemical signal(s) from host plants. As shown in Figure $4 \mathrm{~B}$ and $4 \mathrm{C}$, when the plant extract was present in EMSA, the binding between XerR and the $x c c R$ promoter was disrupted in a dose-dependent manner. We found that the expression of xerR was increased in planta, and XerR-mediated upregulation of the transcription level of $X C 3756$ was not affected when $X c c$ was grown in the host plant, implicating that the unidentified plant chemical(s) has a specific influence on the binding between XerR and the $x c c R$ promoter. Inter-kingdom communications involved in de-repressing the expression of a bacterial gene by plant signal(s) also occur in other bacterial-eukaryotic systems. For instance, the repression of pectinase genes by the transcriptional repressor $\mathrm{KdgR}$ in Erwinia carotovora was abolished in the presence of plant cell wall breakdown products, and agrocinopines de-repressed the AccR binding activity to the arc operon in A. tumefaciens [4648]. Intriguingly, the same plant extract improved the binding of XccR to the luxXc box of the pip promoter, indicating that XerR and XccR may simultaneously interplay with plant signal(s) in regulation of the $x c c R / p i p$ locus, imposing a strict control on the expression of a virulence gene.

It was shown that host plant signal(s) with molecular weights less than $1 \mathrm{kDa}$ inhibited the repression function of XerR in a concentration-dependent manner (Figure 4B and $4 \mathrm{C}$ ). Although most of the small signaling molecules to date were extracted by organic solvents, including furanones, flavonoid, riboflavin and its derivative lu- 
michrome [49-52], our cabbage extract was water based. We propose that the signal(s) may be peptides, amino acid or its derivatives, monosaccharides, oligosaccharides, aminosugars, aminoglycosides or acid and alkali compounds. It has been reported that two free amino acids, homoserine and asparagine, act as host signals inducing the pelD expression of Nectria haematococca in pea seedlings [53].

In brief, in the interaction between $X c c$ and the host plant, $X c c$ builds up a sophisticated mechanism in the $x e r R / x c c R / p i p$ pathway. In this pathway plant signal(s) either activates XccR to positively regulate pip transcription or relieves the inhibition of XerR on $x c c R$ expression. As illustrated in Figure 6, we propose a model that the transcriptional inhibitory role of XerR is regulated by an unknown HK, and XerR then blocks the transcription of $x c c R$ by directly binding to the $x c c R$ upstream sequence during growth in the medium. When $X c c$ grows in the host plant, the conformational changes of XerR and XccR induced by the plant small molecule(s) lead to the release of XerR from the $x c c R$ promoter and the increase in the binding of XccR to the luxXc box. Consequently, the expression of the pip gene, which plays a crucial role in bacterial pathogenesis, is fine-tuned in the host plant.

\section{Materials and Methods}

\section{Strains and reagents}

The wild-type Xcc strain 8004 and Xcc 8177 harboring a chro- mosomal $x c c R$-P/gusA fusion in Xcc 8004 were described previously $[20,54] . X c c$ strains were routinely cultivated at $28{ }^{\circ} \mathrm{C}$ in NYG medium, whereas $E$. coli strains were grown aerobically at $37{ }^{\circ} \mathrm{C}$ in LB medium. Antibiotics were added at the following concentrations: for $X c c$, rifampicin $(100 \mu \mathrm{g} / \mathrm{ml})$, spectinomycin $(150 \mu \mathrm{g} / \mathrm{ml})$ and kanamycin $(100 \mu \mathrm{g} / \mathrm{ml})$; for $E$. coli, spectinomycin $(150 \mu \mathrm{g} / \mathrm{ml})$, kanamycin $(50 \mu \mathrm{g} / \mathrm{ml})$ and ampicillin $(100 \mu \mathrm{g} /$ $\mathrm{ml})$. The reagents 5-bromo-4-chloro-3-indolyl- $\beta$-D-glucuronic acid (X-Gluc), 4-methylumbelliferyl- $\beta$-D-glucuronide (MUG), 4-methyl-umberlliferone and lithium potassium acetyl phosphate were purchased from Sigma. RNase-free DNase, M-MLV Reverse Transcriptase and Random Primers were from Promega and the Lightshift Chemiluminescent EMSA kit was from Pierce.

\section{Creation of Xcc mutants and preparation of in trans expres-} sion constructs

Deletion strains were generated with the suicide vector $\mathrm{pK}$ $18 \mathrm{mobSacB}$ [55] by a long-flanking homology procedure and twostep recombination [56]. All DNA manipulations were performed according to standard procedures. Plasmid DNA was transferred to E. coli by heat shock and to Xcc strains by electroporation. Unless otherwise specified, the corresponding gene fragments were PCR amplified and first cloned into pEASY-T1 Vector (TransGen Biotech) for sequence verification. After digestion with appropriate enzymes, these fragments were cloned into corresponding vectors to generate the constructs used in this study.

To construct Xcc 8098, two 500-bp sequences upstream and downstream of the xer $R$ reading frame were amplified by PCR. The in-frame deletion resulted in removal of the codons for amino acid residues 8 to 428 . After digestion with appropriate enzymes, a pK18xerR clone was created by cloning two recovered fragments into $\mathrm{pK} 18$ mobSacB simultaneously. The $\mathrm{pK} 18 x e r R$ plasmid conferring kanamycin resistance $\left(\mathrm{Kan}^{\mathrm{R}}\right)$ and sucrose sensitivity $\left(\mathrm{Suc}^{\mathrm{S}}\right)$ from white colonies was verified by restriction digestions

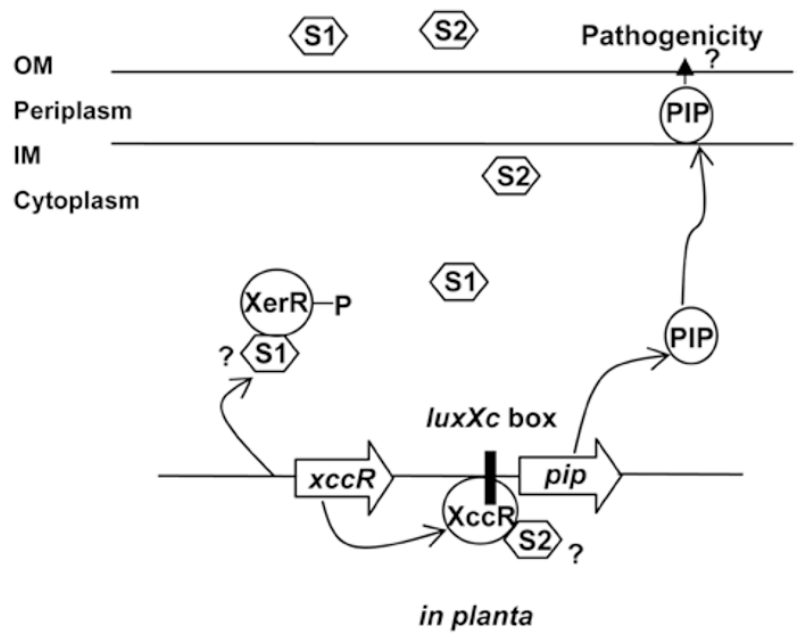

Figure $6 \mathrm{~A}$ model for expression regulation of $x c c R / p i p$ locus by XerR. When $X c c$ is grown in NYG medium, XerR represses the expression of $x c c R$ and pip. The activity of XerR is dependent on the phosphorylation by an unknown two-component signaling transduction system. After entering the host plant, XerR relieves its inhibition to $x c c R$ expression in response to specific plant small molecule(s). The released $X_{c c R}$ binds to lux $X c$ box in the presence of the same or different plant signal(s) to induce the transcription of the pip gene for bacterial virulence. S1 and S2 denote the possible signal(s) from the host. OM and IM refer to the outside and inside membrane, respectively. 
or by sequencing, and then transferred to Xcc. Allelic replacement was achieved by sequential selections on kanamycin and $10 \%$ sucrose to create $X c c 8098$. Positive transformants of Xcc 8098 were confirmed by PCR and sequencing. To introduce $x c c R-\mathrm{P} / g u s A$ into $X c c 8098$, a 3.2-Kb DNA fragment carrying the $x c c R$ promoter and the gusA gene was cloned into pK18mob vector to generate plasmid pFR435. The suicide vector pFR435 was integrated into the chromosome of Xcc 8098 by homologs recombination via a 542bp sequence of the $x c c R$ promoter. The resultant strain was termed Xcc 8099 . PCR was used to identify positive transformants, and the PCR products were sequenced. The same procedures were applied in generation of the other two deletion mutants: xerR $\Delta \mathrm{RR}$ and $x e r R \Delta \mathrm{HTH}$.

To perform in trans expression analyses, the entire coding sequence of the $x e r R$ gene was PCR-amplified and constructed into pHM1, a broad host range expressing plasmid [57]. The generated clone was sequence verified and named pFR423. pFR423 was then electrotransformed into xerR null mutant 8098, 8099 and Xcc 8004 , resulting in the complemented and overexpressed strains Xcc 8098/pFR423, Xcc 8099/pFR423 and Xcc 8004/pFR423, respectively. The resultant transformants were selected on NYG medium supplemented with rifampicin and spectinomycin. Plasmid derivatives harboring the correct inserts in Xcc strains were extracted and verified by restriction digestions.

\section{Site-directed mutagenesis}

Three conserved amino acid residues of XerR (Asp-17, Asp60 and Phe-106, in the RR domain) predicted to be involved in phosphorylation were identified via the sequence alignment with the homologs: NtrC (NCBI accession number X85104), CheY (M13463) and LuxO (L26221). A 753-bp fragment containing part of the $x e r R$ gene and its flanking sequence was PCR-amplified and cloned into pEASY-T1, resulting in pEASY-T753. Three conserved residues were changed to lysine (D17K), alanine (D60A), glutamate (D60E) and tryptophan (F106W) by site-directed mutagenesis (Easy Mutagenesis Systems, TransGen Biotech) using the pEASY-T753 vector as the template. The four resultant 753bp mutant fragments were separately inserted via SpeI and $P v u \mathrm{I}$ sites into pK18xerR-28, a plasmid containing the xerR gene and its up- and down-stream flanking sequences in $\mathrm{pK} 18 \mathrm{mobSacB}$. By homologs recombinations, the mutation-containing plasmids pK18xerR-D17K, pK18xerR-D60A, pK18xerR-D60E and pK18xerR-F106W were individually incorporated into Xcc 8098. Positive clones were verified by PCR and DNA sequencing. The same homologs recombination procedures were used to insert the $x c c R-\mathrm{P} / g$ us $A$ cassette into different mutants to create $x e r R \mathrm{D} 17 \mathrm{~K}$, $x e r R$ D60A, xerR D60E and xerR F106W.

\section{GUS assay}

GUS assays were used to examine the expression of the $x c c R$ $\mathrm{P} / g u s A$ fusion in different $X c c$ strains. GUS activity of the bacteria grown in medium and in planta was measured by the fluorometric method using MUG as a substrate essentially as described in Zhang et al. [20].

\section{$R N A$ extraction and RT-PCR analysis}

Bacterial cells at $\mathrm{OD}_{600}$ of 1.5 to 2.0 were harvested by centrifugation at $4{ }^{\circ} \mathrm{C}$ for $2 \mathrm{~min}$ at $12000 \times \mathrm{g}$. Total bacterial RNA was isolated using TRIzol reagent (Invitrogen) following the protocol provided by the manufacturer. RNase-free DNase I was used to treat the RNA samples. RT was performed using M-MLV Reverse Transcriptase with random hexadeoxynucleotides as primers. Typically, $25 \mathrm{ng}$ of cDNAs was used for each PCR reaction in a $25 \mu \mathrm{l}$ mixture. The 16S rRNA was served as an RT-PCR internal reference.

To assay the level of the pip transcript in Xcc strains grown in planta, Xcc cells were vacuum infiltrated into cabbage seedlings as described by Zhang et al. [20]. Plant leaves harvested at $30 \mathrm{~h}$ post infiltration were homogenized in liquid nitrogen, and RNA isolation and RT-PCR were performed as described above. RNA from un-infiltrated leaves was used as a control.

\section{Protein expression and purification}

Prokaryotic expression plasmids pMX766 (pMal-p2X(lac-P/ xerR)), pMX767 (pMal-p2X(lac-P/xerR-D60A)), pMX768 (pMal$\mathrm{p} 2 \mathrm{X}($ lac-P/xerR-D60E)), pMX769 (pMal-p2X(lac-P/xccR)) were transformed into $E$. coli TB1. Two milliliters of the overnight culture were inoculated into $200 \mathrm{ml} \mathrm{LB}$ broth plus $2 \%$ glucose and ampicillin. After the cells were grown at $37^{\circ} \mathrm{C}$ to an $\mathrm{OD}_{600}$ of 0.5 , MBP-fusion proteins were induced by addition of IPTG to a final concentration of $0.3 \mathrm{mM}$ and cell growth was continued overnight at $16{ }^{\circ} \mathrm{C}$ with a gentle shaking at 180 r.p.m. The cells were harvested, and the soluble MBP-tagged proteins were purified by affinity chromatography with amylose resin (BioLabs). Briefly, each cell pellet was resuspended in $10 \mathrm{ml}$ of the column buffer (20 $\mathrm{mM}$ Tris- $\mathrm{HCl}, \mathrm{pH} 8.0 ; 200 \mathrm{mM} \mathrm{NaCl} ; 1 \mathrm{mM}$ EDTA) plus $1 \mathrm{mM}$ PMSF, incubated on ice for $20 \mathrm{~min}$, sonicated, centrifuged and the supernatant was added to $2 \mathrm{ml}$ of amylose resin slurry. After washing six times with the column buffer, the proteins were eluted using $10 \mathrm{ml}$ of the column buffer plus $10 \mathrm{mM}$ maltose three times. The purified protein samples were combined, and the solvent was changed to a buffer containing $10 \mathrm{mM}$ Tris- $\mathrm{HCl}(\mathrm{pH} 7.5), 50 \mathrm{mM}$ $\mathrm{KCl}, 1 \mathrm{mM}$ DTT, 5\% glycerol. Finally, the proteins were concentrated to approximately $1-10 \mathrm{mg} / \mathrm{ml}$ using Amicon YM-10 column (Millipore) and filtered through an Ultrafree-MC $(0.45 \mu \mathrm{m})$ spin filter (Millipore) before aliquoting for storage at $-80{ }^{\circ} \mathrm{C}$. Protein concentrations were measured using the Bio-Rad Protein Assay reagent with BSA as a standard. About $5 \mu \mathrm{g}$ of each protein sample was analyzed by $8 \%$ SDS-PAGE to verify molecular weight and purity.

\section{Electrophoretic mobility shift assay}

MBP-XerR and MBP-XccR fusion proteins were purified through amylose columns as described above. Four 59-nt singlestranded DNA oligonucleotides containing putative XerR-binding sequences upstream of the $x c c R$ coding region and two 46-nt pip promoter sequences with or without biotin labeling were synthesized by Invitrogen. DNA duplexes required for EMSA were annealed by mixing equal amounts of single-stranded oligos and incubating the mixture for $10 \mathrm{~min}$ at $93{ }^{\circ} \mathrm{C}$ in annealing buffer $(10$ $\mathrm{mM}$ Tris- $\mathrm{HCl}, \mathrm{pH} 7.5 ; 1 \mathrm{mM}$ EDTA; $100 \mathrm{mM} \mathrm{NaCl})$. After slowly cooling down for $2 \mathrm{~h}$ at room temperature, the annealed probes were aliquoted for storage at $-20{ }^{\circ} \mathrm{C}$ and thawed on ice before use. The isotope-labeled probe was end-labeled by using $\left(\alpha-{ }^{32} \mathrm{P}\right)$-dATP (PerkinElmer) and the Klenow fragment of DNA polymerase I (Promega). The labeled probe was purified with Sephadex G-50.

Binding reaction mixtures contained $20 \mathrm{fmol}$ of the DNA biotin-labeled probe, various amounts of MBP-XerR or MBP-XccR 
protein in a buffer of $10 \mathrm{mM}$ Tris- $\mathrm{HCl}(\mathrm{pH} 7.5), 50 \mathrm{mM} \mathrm{KCl}, 1$ $\mathrm{mM}$ DTT, $50 \mathrm{ng} / \mu \mathrm{l}$ poly(dI-dC) DNA in a volume of $20 \mu \mathrm{l}$. After a 30 -min incubation at room temperature, $4 \mu 1$ of $80 \%$ glycerol was added to each reaction and samples were size fractionated using $5 \%$ polyacrylamide gels in $0.5 \times \mathrm{TBE}$ buffer ( $45 \mathrm{mM}$ Tris-borate; $1 \mathrm{mM}$ EDTA) at $4{ }^{\circ} \mathrm{C}$. For competition, a certain amount of unlabeled probe or plant signal(s) was co-incubated with the protein for $20 \mathrm{~min}$ at room temperature before adding labeled probe. The reaction samples were electrophoretically transferred to a nylon membrane (Hybond- $\mathrm{N}^{+}$, Amersham Biosciences) using wet transfer, and then the membranes were crosslinked by a UV lamp at $120 \mathrm{~mJ} / \mathrm{cm}^{2}$. Detection of biotin-activated light signals was performed according to the manufacturer's instructions described by the LightShift Chemiluminescent EMSA Kit (Pierce).

For EMSA using isotope-labeled probe, 8 fmol of labeled probe was added to the mixtures. The reaction procedure and electrophoresis were the same as described above. The gel was dried and subjected to autoradiography.

\section{Phosphorylation of MBP-XerR protein}

Phosphorylation of the purified MBP-XerR protein was performed essentially as described previously [58, 59]. Briefly, 50 $\mu \mathrm{g}$ of MBP-XerR was incubated with $50 \mathrm{mM}$ acetyl phosphate (lithium, potassium salt, from Sigma) for $1 \mathrm{~h}$ at $30{ }^{\circ} \mathrm{C}$ in a buffer of $100 \mathrm{mM}$ Tris-HC1 (pH 7.4), $10 \mathrm{mM} \mathrm{MgC1}{ }_{2}, 125 \mathrm{mM} \mathrm{KC1}$. The concentration of the phosphorylated protein was measured, and the conditions for using it in EMSA were the same as described above. In parallel, similar reactions lacking acetyl phosphate were used to prepare MBP-XerR for EMSA studies.

\section{Preparation of low-molecular-weight plant extracts}

About $20 \mathrm{~g}$ fresh cabbage leaves were homogenized in liquid nitrogen, and the powder was resuspended in $100 \mathrm{ml}$ water. The extract was centrifuged, fractionated in series by $0.45 \mu \mathrm{m}$ filter membrane, ultrafiltration membranes YM10 and YM1 to obtain the compounds of molecular weights less than $1 \mathrm{kDa}$.

\section{Acknowledgments}

This work was supported by grants from the National Natural Science Foundation of China (No 31030008 and No 30471135), and the National Basic Research Program of China (2011CB100700).

\section{References}

1 Decho AW, Norman RS, Visscher PT. Quorum sensing in natural environments: emerging views from microbial mats. Trends Microbiol 2010; 18:73-80.

2 Mullard A. Microbiology: Tinker, bacteria, eukaryote, spy. Nature 2009; 459:159-161.

3 Antunes LC, Ferreira RB. Intercellular communication in bacteria. Crit Rev Microbiol 2009; 35:69-80.

4 Engebrecht J, Nealson K, Silverman M. Bacterial bioluminescence: isolation and genetic analysis of functions from Vibrio fischeri. Cell 1983; 32:773-781.

5 Miller MB, Bassler BL. Quorum sensing in bacteria. Annu Rev Microbiol 2001; 55:165-199.
6 Waters CM, Bassler BL. Quorum sensing: cell-to-cell communication in bacteria. Annu Rev Cell Dev Biol 2005; 21:319. 346.

7 Bassler BL, Losick R. Bacterially speaking. Cell 2006; 125:237-246

8 Ng WL, Bassler BL. Bacterial quorum-sensing network architectures. Annu Rev Genet 2009; 43:197-222.

9 Cao H, Yang M, Zheng H, et al. Complex quorum-sensing regulatory systems regulate bacterial growth and symbiotic nodulation in Mesorhizobium tianshanense. Arch Microbiol 2009; 191:283-289.

10 Chevrot R, Rosen R, Haudecoeur E, et al. GABA controls the level of quorum-sensing signal in Agrobacterium tumefaciens. Proc Natl Acad Sci USA 2006; 103:7460-7464.

11 Haudecoeur E, Planamente S, Cirou A, et al. Proline antagonizes GABA-induced quenching of quorum-sensing in Agrobacterium tumefaciens. Proc Natl Acad Sci USA 2009; 106:14587-14592.

12 Case RJ, Labbate M, Kjelleberg S. AHL-driven quorumsensing circuits: their frequency and function among the Proteobacteria. ISME J 2008; 2:345-349.

13 Subramoni S, Venturi V. LuxR-family 'solos': bachelor sensors/regulators of signalling molecules. Microbiology 2009; 155:1377-1385.

14 Patankar AV, Gonzalez JE. Orphan LuxR regulators of quorum sensing. FEMS Microbiol Rev 2009; 33:739-756.

15 Lequette Y, Lee JH, Ledgham F, Lazdunski A, Greenberg EP. A distinct QscR regulon in the Pseudomonas aeruginosa quorum-sensing circuit. J Bacteriol 2006; 188:3365-3370.

16 Danino VE, Wilkinson A, Edwards A, Downie JA. Recipientinduced transfer of the symbiotic plasmid pRL1JI in Rhizobium leguminosarum bv. viciae is regulated by a quorumsensing relay. Mol Microbiol 2003; 50:511-525.

17 McIntosh M, Krol E, Becker A. Competitive and cooperative effects in quorum-sensing-regulated galactoglucan biosynthesis in Sinorhizobium meliloti. J Bacteriol 2008; 190:53085317.

18 Ahmer BM. Cell-to-cell signalling in Escherichia coli and Salmonella enterica. Mol Microbiol 2004; 52:933-945.

19 Ryan RP, Dow JM. Diffusible signals and interspecies communication in bacteria. Microbiology 2008; 154:1845-1858.

20 Zhang L, Jia Y, Wang L, Fang R. A proline iminopeptidase gene upregulated in planta by a LuxR homolog is essential for pathogenicity of Xanthomonas campestris pv. campestris. Mol Microbiol 2007; 65:121-136.

21 Ferluga S, Venturi V. OryR is a LuxR-family protein involved in interkingdom signaling between pathogenic Xanthomonas oryzae pv. oryzae and rice. J Bacteriol 2009; 191:890-897.

22 Ferluga S, Bigirimana J, Hofte M, Venturi V. A LuxR homologue of Xanthomonas oryzae pv. oryzae is required for optimal rice virulence. Mol Plant Pathol 2007; 8:529-538.

23 De Carlo S, Chen B, Hoover TR, et al. The structural basis for regulated assembly and function of the transcriptional activator NtrC. Genes Dev 2006; 20:1485-1495.

24 Kim HS, Lee MA, Chun SJ, Park SJ, Lee KH. Role of NtrC in biofilm formation via controlling expression of the gene encoding an ADP-glycero-manno-heptose-6-epimerase in the pathogenic bacterium, Vibrio vulnificus. Mol Microbiol 2007; 63:559-574. 
25 Freeman JA, Bassler BL. A genetic analysis of the function of LuxO, a two-component response regulator involved in quorum sensing in Vibrio harveyi. Mol Microbiol 1999; 31:665-677.

26 Andrade MO, Alegria MC, Guzzo CR, et al. The HD-GYP domain of RpfG mediates a direct linkage between the Rpf quorum-sensing pathway and a subset of diguanylate cyclase proteins in the phytopathogen Xanthomonas axonopodis pv citri. Mol Microbiol 2006; 62:537-551.

27 Stock AM, Robinson VL, Goudreau PN. Two-component signal transduction. Annu Rev Biochem 2000; 69:183-215.

28 Zhu X, Amsler CD, Volz K, Matsumura P. Tyrosine 106 of CheY plays an important role in chemotaxis signal transduction in Escherichia coli. J Bacteriol 1996; 178:4208-4215.

29 Flashner Y, Weiss DS, Keener J, Kustu S. Constitutive forms of the enhancer-binding protein NtrC: evidence that essential oligomerization determinants lie in the central activation domain. J Mol Biol 1995; 249:700-713.

30 Skerker JM, Perchuk BS, Siryaporn A, et al. Rewiring the specificity of two-component signal transduction systems. Cell 2008; 133:1043-1054.

31 Lee SY, De La Torre A, Yan D, et al. Regulation of the transcriptional activator NtrC1: structural studies of the regulatory and AAA+ ATPase domains. Genes Dev 2003; 17:2552-2563.

32 Rojo F. Mechanisms of transcriptional repression. Curr Opin Microbiol 2001; 4:145-151.

33 Rojo F. Repression of transcription initiation in bacteria. $J$ Bacteriol 1999; 181:2987-2991.

34 Parker CT, Sperandio V. Cell-to-cell signalling during pathogenesis. Cell Microbiol 2009; 11:363-369.

35 Von Bodman SB, Bauer WD, Coplin DL. Quorum sensing in plant-pathogenic bacteria. Annu Rev Phytopathol 2003; 41:455-482.

36 Studholme DJ, Dixon R. Domain architectures of $\sigma^{54}$ dependent transcriptional activators. J Bacteriol 2003; 185: $1757-1767$.

37 Kern D, Volkman BF, Luginbuhl P, et al. Structure of a transiently phosphorylated switch in bacterial signal transduction. Nature 1999; 402:894-898.

38 Wyman C, Rombel I, North AK, Bustamante C, Kustu S. Unusual oligomerization required for activity of NtrC, a bacterial enhancer-binding protein. Science 1997; 275:1658-1661.

39 Moore JB, Shiau SP, Reitzer LJ. Alterations of highly conserved residues in the regulatory domain of nitrogen regulator I (NtrC) of Escherichia coli. J Bacteriol 1993; 175:26922701.

40 Rombel I, North A, Hwang I, Wyman C, Kustu S. The bacterial enhancer-binding protein $\mathrm{NtrC}$ as a molecular machine. Cold Spring Harb Symp Quant Biol 1998; 63:157-166.

41 Boehr DD. During transitions proteins make fleeting bonds. Cell 2009; 139:1049-1051.

42 Gardino AK, Villali J, Kivenson A, et al. Transient non-native hydrogen bonds promote activation of a signaling protein. Cell 2009; 139:1109-1118.

43 Kenney LJ. How important is the phosphatase activity of sensor kinases? Curr Opin Microbiol 2010; 13:168-176.

44 Silversmith RE. Auxiliary phosphatases in two-component signal transduction. Curr Opin Microbiol 2010; 13:177-183.
45 Porter SL, Roberts MA, Manning CS, Armitage JP. A bifunctional kinase-phosphatase in bacterial chemotaxis. Proc Natl Acad Sci USA 2008; 105:18531-18536.

46 Newton JA, Fray RG. Integration of environmental and hostderived signals with quorum sensing during plant-microbe interactions. Cell Microbiol 2004; 6:213-224.

47 Piper KR, Beck Von Bodman S, Hwang I, Farrand SK. Hierarchical gene regulatory systems arising from fortuitous gene associations: controlling quorum sensing by the opine regulon in Agrobacterium. Mol Microbiol 1999; 32:1077-1089.

48 Liu Y, Jiang G, Cui Y, et al. $k d g R_{E c c}$ negatively regulates genes for pectinases, cellulase, protease, harpin ${ }_{\mathrm{Ecc}}$, and a global RNA regulator in Erwinia carotovora subsp. carotovora. $J$ Bacteriol 1999; 181:2411-2421.

49 Straight PD, Kolter R. Interspecies chemical communication in bacterial development. Annu Rev Microbiol 2009; 63:99118.

50 Manefield M, Rasmussen TB, Henzter M, et al. Halogenated furanones inhibit quorum sensing through accelerated LuxR turnover. Microbiology 2002; 148:1119-1127.

51 Rajamani S, Bauer WD, Robinson JB, et al. The vitamin riboflavin and its derivative lumichrome activate the LasR bacterial quorum-sensing receptor. Mol Plant Microbe Interact 2008; 21:1184-1192.

52 Soto MJ, Dominguez-Ferreras A, Perez-Mendoza D, Sanjuan J, Olivares J. Mutualism versus pathogenesis: the giveand-take in plant-bacteria interactions. Cell Microbiol 2009; 11:381-388.

53 Yang Z, Rogers LM, Song Y, Guo W, Kolattukudy PE. Homoserine and asparagine are host signals that trigger in planta expression of a pathogenesis gene in Nectria haematococca. Proc Natl Acad Sci USA 2005; 102:4197-4202.

54 Turner P, Barber C, Daniels M. Behavior of the transposons $\mathrm{Tn} 5$ and Tn7 in Xanthomonas campestris pv. campestris. Mol Gen Genet 1984; 195:101-107.

55 Schafer A, Tauch A, Jager W, et al. Small mobilizable multipurpose cloning vectors derived from the Escherichia coli plasmids pK18 and pK19: selection of defined deletions in the chromosome of Corynebacterium glutamicum. Gene 1994; 145:69-73.

56 Skerker JM, Prasol MS, Perchuk BS, Biondi EG, Laub MT. Two-component signal transduction pathways regulating growth and cell cycle progression in a bacterium: a systemlevel analysis. PLoS Biol 2005; 3:e334.

57 Innes RW, Hirose MA, Kuempel PL. Induction of nitrogenfixing nodules on clover requires only 32 kilobase pairs of DNA from the Rhizobium trifolii symbiosis plasmid. J Bacteriol 1988; 170:3793-3802.

58 Pogliano J, Lynch AS, Belin D, Lin EC, Beckwith J. Regulation of Escherichia coli cell envelope proteins involved in protein folding and degradation by the Cpx two-component system. Genes Dev 1997; 11:1169-1182.

59 Lynch AS, Lin EC. Transcriptional control mediated by the ArcA two-component response regulator protein of Escherichia coli: characterization of DNA binding at target promoters. J Bacteriol 1996; 178:6238-6249. 